\title{
Design of Coin Sorter
}

\author{
Li Feng ${ }^{1, ~ a}$, Ning Tingzhou ${ }^{2, b, ~ * ~}$ \\ ${ }^{1}$ School of Mechanical Engineering, Shandong Huayu University of Technology, Dezhou, 253034, China \\ ${ }^{2}$ College of Mechanical and Electrical Engineering, Zaozhuang University, Zaozhuang, 277160, China \\ alwfjh@163.com, bning-tingzhou@163.com \\ *corresponding author
}

Keywords: false identification system; single chip microcomputer; liquid crystal display

\begin{abstract}
Based on the status quo of coin sorting machines at home and abroad, a coin sorting machine with false identification function was designed. The coin enters the entrance of the coin, through the rotation of the coin disk and the flattening of the pressing plate, a single coin enters the coin hole of the coin disk, then passes through the false identification device, passes through the four branches arranged in order from small to large, and falls into the collection device of corresponding value respectively. The coin exit is installed with photoelectric counting device, which counts the coins of each denomination and integrates the total amount to be displayed on the display screen through the operation of single chip microcomputer. The coins of the same denomination will be placed in the same receiving box to be sorted.
\end{abstract}

\section{Introduction}

Foreign coin sorters have developed a long time, and in some developed countries at abroad, the type of coins in circulation is very big[1-2], but its circulation and operation management of the equipment is also very much. According to the technical characteristics, it can be divided into count type, classification type, packaging type, cleaning and disinfection type, exchange type and self-service type. Although the technology of foreign coin sorter is mature, but the price is expensive, and the Chinese currency system is complicated, it is difficult for foreign coin sorter to popularize in China.

China began to introduce relevant products from foreign countries in the mid-1990s. At present, small coin sorters have appeared in China, but there is a general lack of false identification. Therefore, it is of great research value and practical significance to study and develop the automatic coin sorter with high precision, stable performance and perfect function [3].

\section{Design scheme of coin sorter}

The design of the coin sorter will set the coin with different diameters according to the trajectory of centrifugal motion and the size of the coin. At the same time, to save space, the motor spindle will be adopted to drive the rotation of the coin disk to transfer power. The design mainly includes separation device, authenticity identification system and counting system. The separation device is the basic component of the coin sorter, which includes coin disk, disconnector, platen, motor, etc. The authentication system and the counting system are connected to the single chip microcomputer, which controls the operation of the authentication device, separates the counterfeit currency, and then displays the result of counting through the LCD.

\section{Design of separation device}

\subsection{Design of coin disc}

The main function of the sorter is to drive the coin by rotating the coin disc, and then the coin passes through the separator disc to separate. In order to reduce the fault-tolerant rate when passing 
the counterfeit detection device, a ring of coin holes is set on the coin disk. The coin falls into the slot and passes through the false identification system before passing through the disjunction along the fixed route.

To ensure the size of the coin sorter is small, the diameter of the coin disc is $300 \mathrm{~mm}$. The thick base and the coin hole are determined according to the thickness and diameter of different coins, that is, $3 \mathrm{~mm}$ is selected for the thick base and $35 \mathrm{~mm}$ is selected for the token hole. In order to make the holes as large as possible, the distance between each two holes should be as small as possible, and on the basis of the design, the distance between the center of the coin hole and the edge of the coin disk should be determined as $27.5 \mathrm{~mm}$. The rotating speed of the disc is determined by force analysis of the coin. The force analysis of coin is shown in Figure 1.

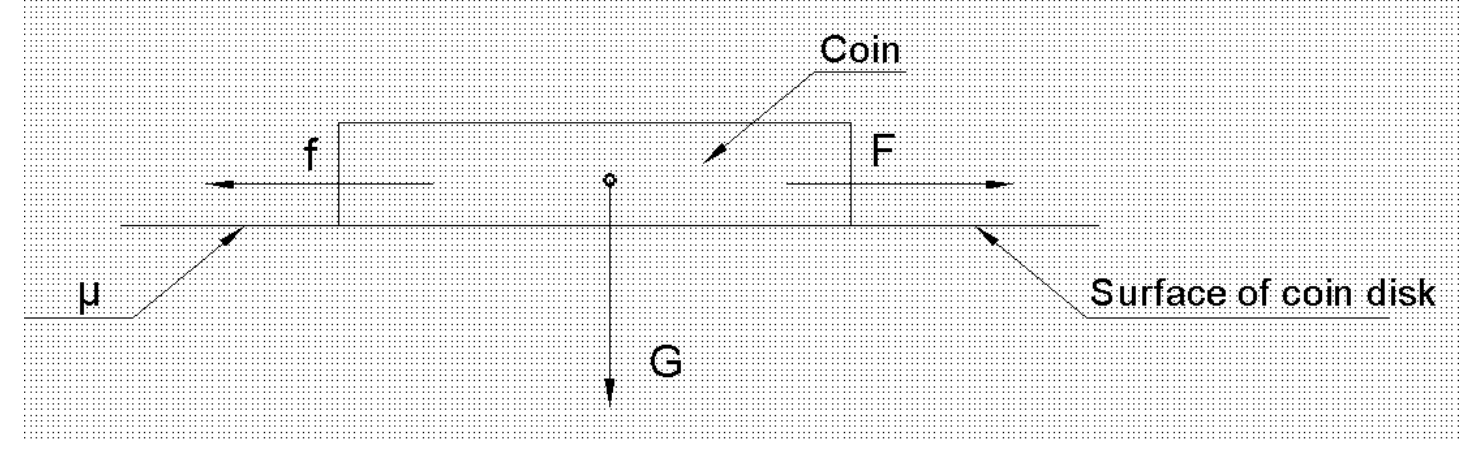

Fig.1 Force analysis of coin

Notes: $\mu$ is the coefficient of friction, $\mathrm{f}$ is the frictional force, $\mathrm{F}$ is the centripetal force, $\mathrm{G}$ is the gravity.

According to Equation,

$$
\begin{gathered}
\mathrm{f}=\mu \mathrm{G}=\mu \mathrm{mg} \\
F=\frac{m V^{2}}{r}=m \omega^{2} r
\end{gathered}
$$

United stand, $F=m \omega^{2} r>F=\mu m g$

$$
\omega>\sqrt{\frac{\mu g}{r}}>0
$$

As the coin radius and the friction coefficient are different, the corresponding values should be obtained according to the mechanical design manual respectively, and then substituted to calculate. Lastly, a maximum value is taken.

$$
\begin{gathered}
\omega>\sqrt{\frac{\mu g}{r}}=\sqrt{\frac{0.30 \times 9.8}{0.019}}=16.059 \mathrm{rad} / \mathrm{s} \\
v=\omega \mathrm{r}=16.059 \times \frac{30}{\pi}=153.35 \mathrm{r} / \mathrm{min}
\end{gathered}
$$

In order to meet the requirement that the centripetal force of the coin is greater than the friction, so the minimum speed required is $153 \mathrm{r} / \mathrm{min}$, and this is the estimated value. Here, $200 \mathrm{r} / \mathrm{min}$ is the speed of the coin disk.

\subsection{Design of separation disc}

There is a row of coins on the separation tray from small to large, the whole sorting machine is dependent on the coin sorting operation. In order to ensure the durability of the separation plate, both the two sides of the coin slot are in the form of semicircle.

The minimum length of the coin slot is calculated by the time the coin falls free from the disc. 
Choose the thickest coin here. According to Equation,

$$
S=\frac{g t^{2}}{2}
$$

Then, $t=\sqrt{\frac{2 S}{g}}=\sqrt{\frac{2 \times 2.4 m m}{9.8 m / s^{2}}}=0.219 \mathrm{~s}$

Linear velocity $v=\omega r=200 \mathrm{r} / \mathrm{min} \times 300 \mathrm{~mm}=\pi \mathrm{m} / \mathrm{s}$

The taken time for the coin to leave the coin disk turns $68.8 \mathrm{~mm}$ with the coin disk, so the minimum length of the coin slot is $68.8 \mathrm{~mm}$. In the form of semicircle on both sides of the coin slot, the arc distance between the center of the coin slot and the data determined is: both the new 1Mao and the $5 \mathrm{Mao}$ are $70 \mathrm{~mm}$, the new and old 1 Yuan are $80 \mathrm{~mm}$, and the old $1 \mathrm{Mao}$ is $90 \mathrm{~mm}$.

\subsection{Design of false identification system}

At present, there are four types of coin identification: weighing, image identification and eddy current identification. On the whole, the device adopts the eddy current sensor because of its high efficiency, low price and no direct contact with coins, which avoids the disadvantages of decreasing the life of the separator caused by wear. Figure 2 shows the schematic diagram of the eddy current sensor.

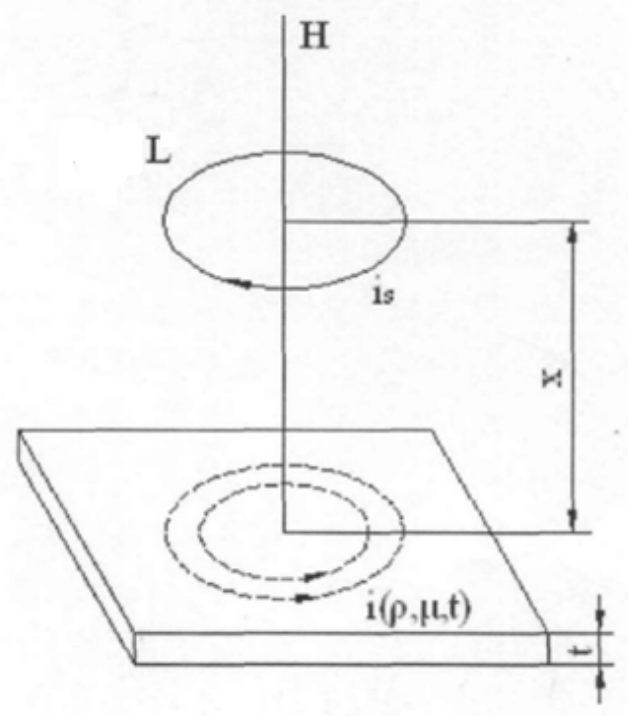

Fig.2 Schematic diagram of electric eddy current sensor

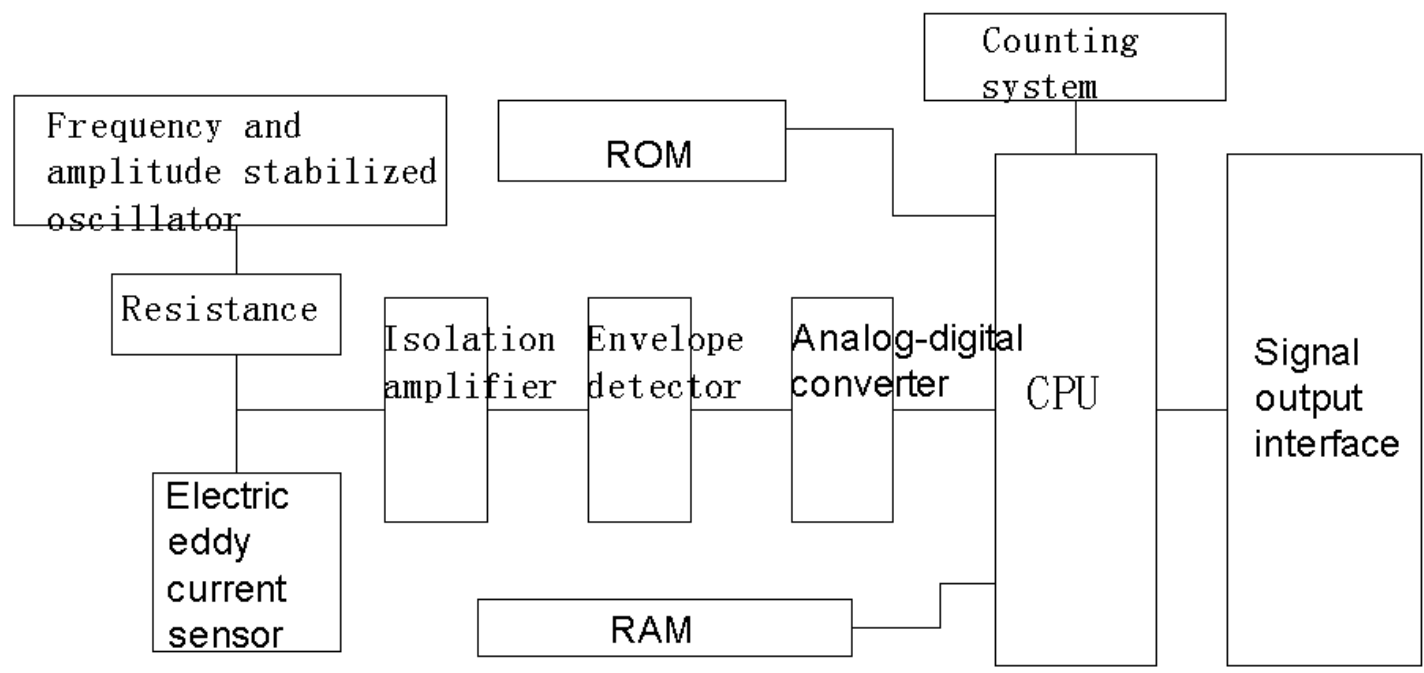

Fig.3 MCU connection diagram 
The working process is as follows: when the coin passes through the eddy current sensor, it can cause different internal oscillation frequencies, so the data is sent to the processing device, and then compared with the real coin to identify the authenticity. The result of the single chip computer is used to control the switch of the counterfeit money collection device so that the counterfeit money falls into the counterfeit money collection device. The work flow is shown in Figure 3.

\subsection{Design of counting device and receiving tray}

Photoelectric counter is an automatic counting device made of photoelectric elements. The design selects the infrared counter tube to realize the statistics of the coin. Connect the infrared laser tube with the microcontroller, and then connect the LCD screen. After the work of the infrared laser tube, the statistical data of the microcontroller is analyzed and calculated, lastly, output to the LCD screen.

The receiving tray can withstand the impact of backward coins, at the same time, the coin can't stay at the edge of the receiving disc due to the high friction, so the minimum angle of the coin should be analyzed according to the force diagram of the coin. The force of the coin is shown in Figure 4.

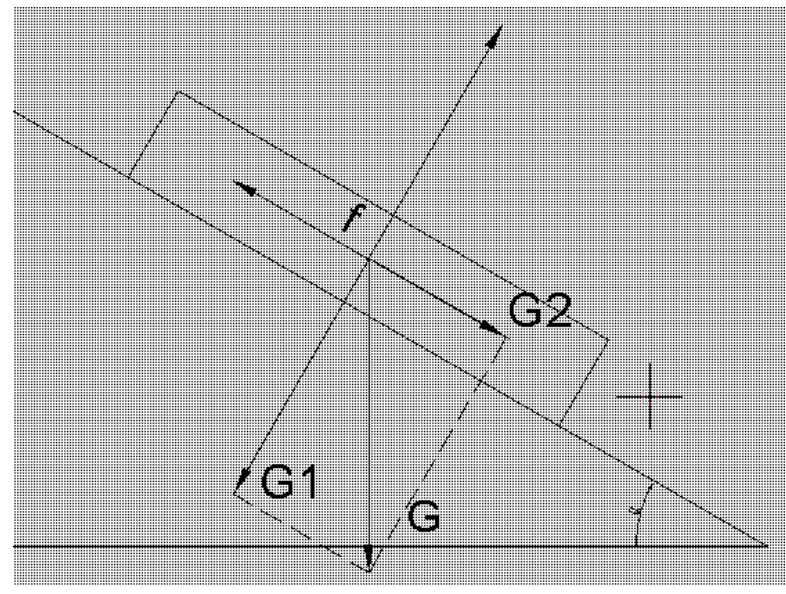

Fig 4. Force diagram of coin

Notes: $\mathrm{f}$ is the frictional force, $\mathrm{G}$ is the gravity.

Where, $G_{2}>f$, it is calculated according to Equation,

$$
\begin{gathered}
f=\mu m g \\
G_{2}=\sin \alpha \times m g
\end{gathered}
$$

United stand, $\sin \alpha>\mu$

Transform available, $\alpha>\arcsin \mu$

The old 1 Mao needs $\alpha \geq 30^{\circ}$, and others just need $\alpha>17.5^{\circ}$. Since the old 1 Mao is less, so choose $\alpha=30^{\circ}$. And two opposite holes need to be punched under the coin plate to install the infrared butt tube.

\section{Conclusion}

The design of coin sorting machine with false detection function is finally realized, which can realize the coin sorting and have the functions of false identification and counting. The counting results will be displayed on the LCD screen through the operation of the single chip microcomputer. The coin sorter is simple in design and easy to operate. It can solve the problem of difficult to recycle coins well in places with large coin circulation, such as bus companies, supermarkets and financial departments. 


\section{Acknowledgements}

This work was supported by the Science and Technology Program of Shandong Higher Education Institutions (NO. J17KA034).

\section{References}

[1] KANG Z.Q., CHENG R.F. and HE Q.M.. (2017) Overlap Track Fusion of Belt Coin Sorting Machine Innovation Design . Machine Design and Research, 5, 68-72.

[2] DING F.J., HONG T.J., and CHEN K.D..(2016) Architecture Design and Manufacturing of Coin Sorters Aim at 5th Edition of RMB. Journal of Anhui Science and Technology University, 5, 71-74.

[3] GAO S.T., XU Y., SANG M.Z.. (2017) A Kind of Multi-functional Coin Sorter. Mechanical Engineer, 6, 60-62. 